\title{
Análisis de la participación ciudadana a través de un pionero modelo participativo en prensa: el proyecto «enlaCe» de $\mathrm{El}$ Correo
}

\author{
Terese Mendiguren Galdospín \\ Universidad del País Vasco / EHU \\ terese.mendiguren@ehu.es \\ Jesús CANGA LAREQUI \\ Universidad del País Vasco / EHU \\ jesus.canga@ehu.es
}

Recibido: 11/07/2011

Aceptado: 18/01/2012

\begin{abstract}
Resumen
Hace tiempo que algunos conceptos como el de periodismo colaborativo o periodismo ciudadano nos llevan a pensar que vivimos en la era de la participación ciudadana en la prensa. Los grandes medios de comunicación en Internet se han ido adaptando paulatinamente a una época en la que la audiencia demanda ser escuchada. Los diarios de papel tampoco se han querido quedar atrás, y no son pocos los que han intentado ampliar sus espacios dedicados a las aportaciones de los lectores. Adaptar su modelo a los nuevos tiempos es sin duda un reto difícil. Un ejemplo premiado, pionero y que ha servido como modelo para otros periódicos es el de El Correo y su sección «enlaCe», en cuyo análisis se centra este artículo.
\end{abstract}

Palabras clave: Participación ciudadana, periódicos, papel, enlaCe, El Correo

\section{Analysis of Citizen Participation by a Pioneer Collaborative Press Model: El Correo Newspaper's «enlaCe» Project}

\begin{abstract}
Long ago some concepts such as collaborative journalism or citizen journalism lead us to think we live in an era of citizen participation in the press. The mass media on the Internet have gradually been adapted to a time when the audience demands to be heard. The paper diaries don't want to fall behind, and some of them have tried to expand its space dedicated to reader's contributions. Adapting their model to changing times is certainly a difficult challenge. We find a pioneer example in El Correo newspaper. Its section enlaCe has been prized and has served as a model for other newspapers. This article focuses in the analysis of citizen participation led by «enlaCe».
\end{abstract}

Keywords: Citizen Participation, Newspapers, Paper, Enlace, El Correo

\section{Referencia normalizada}

MENDIGUREN GALDOSPÍN, Terese y CANGA LAREQUI, Jesús (2012): “Análisis de la participación ciudadana a través de un pionero modelo participativo en prensa: El proyecto "enlaCe" de $\mathrm{El} \mathrm{Co}$ rreo". Estudios sobre el mensaje periodístico. Vol. 18, núm. 1, págs.: 133-146. Madrid, Servicio de Publicaciones de la Universidad Complutense.

Sumario: 1. Introducción. 2. «enlaCe», un modelo participativo reconocido y premiado. 3. Objetivos de la investigación, hipótesis y metodología. 4. Contexto de la implantación de enlaCe en el diario El Correo. 5. Resultados del análisis: Evolución de la participación en «enlaCe». 6. Conclusiones. 7. Referencias bibliográficas.

\section{Introducción}

Las ediciones digitales de los medios tradicionales se han ido transformando en los últimos años para recibir con los brazos abiertos a los ciudadanos, dotándoles de herra- 
mientas de participación que en mayor o menor medida permiten la implicación de la audiencia en los contenidos que ofrecen estas webs. La mayoría de estos medios digitales ofrecen diversas vías de participación, como la opción de publicar fotografías, participar en foros, redactar noticias, formular preguntas, registrar blogs o comentar las noticias. Este tipo de incursiones ciudadana en los medios digitales están siendo ampliamente analizadas en los últimos años (RUIZ et al: 2010; ARMENTIA: 2009; LÓPEZ y FERNÁNDEZ: 2009; MAYORAL: 2009; HOYO y OlMO: 2009; GARCÍA DE TORRES et al: 2009; Meso y PALOmo: 2009). Por otro lado, hoy en día existen diarios digitales que desde el inicio nacen con vocación colaborativa. Entre este tipo de medios cabe mencionar el caso del diario coreano OhmyNews ${ }^{1}$ que durante muchos años fue el máximo exponente del periodismo participativo.

Internet da mucho juego, y la prensa digital se está adaptando a la demanda de participación recibida por parte de la ciudadanía. Esta adaptación es más difícil para los periódicos de papel que no pueden ofrecer enlaces, foros, blogs y en definitiva un espacio casi ilimitado de participación al que se está acostumbrando el usuario. Sus características técnicas como soporte de papel inevitablemente limitan el ámbito de actuación de los mismos. De todas formas, gracias a sus ediciones digitales, hoy en día pueden abastecerse de las aportaciones de los lectores como fuentes de noticias, ya que disponen a través de sus webs de un material extenso de informaciones y fotografías provenientes de los ciudadanos. Además con el correo electrónico el contacto con el lector es más inmediato. Gracias a estas nuevas vías de contacto anteriormente inexistentes, y quizá por la influencia del denominado periodismo ciudadano que nació y se ha desarrollado básicamente en la red, lo cierto es que la participación ciudadana ha ganado presencia en algunos periódicos de papel.

Adaptar su modelo a los nuevos tiempos es sin duda un reto difícil. Algunas cabeceras con mayor éxito que otras han ampliado sus secciones dedicadas a la participación ciudadana. Un ejemplo premiado, pionero y que ha servido como modelo para otros periódicos es el del El Correo ${ }^{2}$ y su sección enlaCe, en cuyo análisis se centra este artículo.

\section{2. «enlaCe», un modelo participativo reconocido y premiado}

El análisis de este modelo de participación ciudadana denominado enlaCe ha sido motivado por su propia naturaleza como ejemplo original y pionero en España. Es cierto que otros diarios también han ampliado en los últimos años sus espacios participativos para ofrecer otras alternativas de colaboración por parte del lector. Hace algunos años la sección de cartas al director era el único viso de incursión ciudadana que se

${ }^{1}$ Durante diez años este diario surcoreano se estableció como el ejemplo más claro y reconocido de lo que se entiende por periodismo colaborativo, ya que un porcentaje amplio de sus noticias estaba redactado y documentado por los propios ciudadanos. A finales del año 2010 tuvo que cesar su actividad como medio de comunicación participativo por problemas de financiación.

2 El Correo, cabecera vizcaína del grupo Vocento, es el diario líder de audiencia en su ámbito de difusión y el séptimo periódico a nivel estatal según la OJD. 
podía percibir en los diarios de papel. Ahora es más habitual encontrarse con encuestas que reflejan la opinión del ciudadano, fotos enviadas por lectores u otra serie de herramientas que pretenden fomentar la interacción.

Sin embargo, hasta el lanzamiento de enlaCe, nunca antes había existido un modelo de participación integral del ciudadano en un periódico de papel. De hecho, se trata del único y primer periódico de papel estatal en recibir un premio internacional (premio de la $\mathrm{WAN}^{3}$ ) por su filosofía de participación y colaboración del lector a lo largo de todo el periódico. La iniciativa enlaCe fue considerada como "la primera experiencia integral de periodismo ciudadano en papel" ${ }^{4}$. Hasta entonces había existido como mucho una página o dos dedicadas al lector, pero no el concepto de periódico tan abierto en todas sus secciones.

De hecho, no se trataba únicamente de una sección creada por y para la ciudadanía. Según los profesionales que trabajaban en El Correo, estábamos ${ }^{5}$ ante una filosofía. Un nuevo planteamiento de cómo se deben hacer las cosas en esta era donde la información fluye rápidamente por las manos, tanto de profesionales como de ciudadanos. La misión de esta nueva herramienta comunicacional e interactiva de $\mathrm{El} \mathrm{Co}$ rreo consistía, en la fecha de su lanzamiento, en garantizar la colaboración del lector a lo largo de todo el periódico, con especial presencia a doble página en la sección que lleva por título enlaCe.

Además, la impulsora del proyecto Montserrat Lluis asegura que El Heraldo de Aragón "hizo un modelo calcado tras pasar dos días en Bilbao". El Virginia Pilot de EEUU también envió a su directora de nuevas operaciones, Marian Anderfuren, para conocer el modelo. Según explica Lluis, la representante de Virginia Pilot expresó "su admiración por el excelente funcionamiento" de enlaCe. Hicieron una sección similar pero más pequeña, de una página diaria. Además, otros responsables de diarios de Argentina, Alemania e incluso de Emiratos Árabes pasaron por la redacción del periódico para conocer la sección.

Otro de los factores por los que consideramos acertada la elección del objeto de estudio es que, en los últimos años, tan significativos a nivel de participación ciudadana en los medios, existe una carencia de investigación y análisis de la participación del

3 El Premio fue recogido en marzo del año 2007 en Washington., en el marco de la Séptima Conferencia y Expo Mundial de Jóvenes Lectores. Según fuentes de El Correo, redactores jefe de diarios de Honduras, Panamá, Francia, Argentina y EEUU mostraron su intención de desplazarse a Bilbao para conocer de primera mano la iniciativa; y también profesionales adscritos a diarios españoles como El País, El Mundo o El Diario de Navarra manifestaron en Washington su admiración hacia enlace valorando entre otras cosas la calidad de las colaboraciones de los lectores.

${ }^{4}$ Véase la noticia “enlace, premio mundial e innovación” disponible en: http://www.elcorreo. com/vizcaya/prensa/20061112/sociedad/enlace-premio-mundial-innovacion_20061112.html

5 Se hace la referencia en pasado porque el proyecto enlaCe nació con esa filosofía, digna de estudio, pero hace un año cesó en su empeño por establecerse como un modelo de participación integral a lo largo de todo el diario, para convertirse en una sección cerrada típica. 
lector en los periódicos de papel. Es cierto que algunos autores se han interesado por la implicación y presencia de los lectores en el contexto del papel en la actualidad, como Lluis PASTOR y su libro Teoría de Cartas al Director. La gestión periodística del público (2009); como el profesor de la Universidad de Navarra Miguel Ángel JIMENO (2009), que propone un interesante repaso de algunas fórmulas participativas que ofrecen los diarios en su excelente artículo Los retos del nuevo periodismo ¿Para qué sacamos ciudadanos en el diario si no pensamos en ellos? o la profesora Ainara LARRONDO (2009) que precisamente ponía su atención en enlaCe, el mismo objeto de estudio del presente artículo. Sin embargo, no existe ningún tipo de análisis que, siguiendo una concreta propuesta metodológica, estudie las características y tendencias de la audiencia participativa en el papel actualmente.

\section{Objetivos de la investigación, hipótesis y metodología}

Son varios los objetivos con los que nace este trabajo. En primer lugar, averiguar qué grado de participación genera enlaCe respecto al modelo anterior de participación ciudadana que ofrecía El Correo. También se pretende analizar comparativamente la participación ciudadana entre los distintos espacios que constituyen la sección enlaCe en los períodos temporales propuestos en la metodología, así como conocer el espacio y la cantidad de aportaciones diarias de lectores que publica enlace.

Las hipótesis establecen varios planteamientos iniciales: La transformación de $E l$ Correo para adaptarse a su modelo pionero de participación ciudadana denominado enlaCe se ha producido de forma paulatina. Esto es, el diario ha vivido una transición estudiada, en la que se han ido introduciendo algunos cambios anteriores al lanzamiento de enlaCe. Además, los temas de interés general donde más participa el lector son los relacionados con su entorno inmediato y, por otro lado, las aportaciones ciudadanas a través de enlaCe fueron ocupando cada vez un espacio mayor implicando así la colaboración de la audiencia en la elaboración del contenido del periódico.

Las aportaciones de autores como BARDIN (1986) o KRIPENDORFF (1990) han servido de referente en la metodología planteada en esta investigación. Tal y como apunta Laurence BARDIN, en todo método analítico es fundamental una primera fase de organización. Se trata de un preanálisis que corresponde a un período de intuiciones. Tiene como objetivo "la operalización y la sistematización de las ideas de partida para poder llegar a un sistema preciso de desarrollo de las operaciones sucesivas" (BARDIN, 1986: 71). La fase del análisis es "larga y fastidiosa; consiste esencialmente en operaciones de codificación, descomposición o enumeración en función de consignas formuladas previamente"(BARDIN, 1986: 76). Dicho análisis se ha llevado a cabo a través de una ficha elaborada expresamente para esta investigación. Cada lector, o mejor dicho, la aportación que cada lector realiza en el diario, es considerada una unidad analizable. Es necesario apuntar que las "cartas al director", que ya existían antes de la aparición de enlaCe y no forman parte de esta sección tras su implantación, también se contabilizan en este análisis como un tipo más de aportación ciudadana. Se entiende que es uno de los fundamentales pilares de la participación de los lectores en los diarios de papel. 
Las aportaciones del lector se han clasificado en cinco grandes grupos según contenido:

1. Interés general

2. Ámbito privado o personal

3. Información de servicio

4. Aportación privada de interés general ${ }^{6}$

5. Aportaciones enmarcadas en el pasatiempo y ocio, como las respuestas de los lectores a concursos y retos planteados por parte del diario.

Algunos de estos grupos se dividen a su vez en diferentes subtipos según el contenido y naturaleza de las aportaciones. La ficha contempla otra serie de variables, como la ubicación de las aportaciones (Portada, Ciudadanos, enlaCe, Opinión, Política, Economía, Bolsa, Sociedad, Mundo, Cultura, Agenda, Televisión, Deportes o Gastronomía), la opción de los lectores de obtener algún tipo de compensación ${ }^{7}$ o regalo y el tipo de signo ${ }^{8}$.

Estas categorías son aplicables a cualquier periódico, si bien sus variables pueden ser modificadas o adaptadas. En cuanto al espacio que ocupa cada aportación, no se trata tanto de analizar cuántos centímetros ocupa cada una, sino de saber el espacio que ocupan las aportaciones de los lectores en relación al espacio general del diario, así como de extraer datos que permitan realizar una comparativa del espacio dedicado a diferentes tipos de aportaciones. Por lo tanto, se ha medido el número de módulos que ocupa cada una de las aportaciones. El referente general es que El Correo se divide en 50 módulos por página y publica una media de 86 páginas por ejemplar, con lo que el espacio diario en módulos alcanza la cifra de 4.300.

Tras consultar las opciones de muestreo no probabilístico propuestas por varios autores (BABBIE, 1999; VISAUTA, 1989; COCHRAM, 1971) se decidió que los objetivos planteados por esta tesis encontrarían su mayor grado de respuesta en una metodología analítica basada en un muestreo no probabilístico discrecional y para decidir los períodos temporales que se iban a analizar se ha tenido en cuenta la fecha de lanza-

${ }^{6}$ En esta categoría se clasifican las aportaciones ciudadanas que inicialmente presentan casos de interés privado pero que son lanzadas y publicadas por el diario a modo de interés general, por su planteamiento o repercusión social-coyuntural (p.e. un reportaje sobre un niña cuyo sueño es nadar con delfines. El periódico cumple su sueño, muestra sus reacciones y detalla los cuidados y labores que requieren estos animales)

7 Según el tipo de participación que realicen, los lectores pueden recibir alguna compensación, normalmente a través de sorteos. Todas las herramientas participativas que enlaCe pone a disposición de la audiencia no garantizan necesariamente que los lectores entren en algún sorteo, ni que obtengan regalos. La ficha distingue varias variables bajo el epígrafe "compensación y regalos", que se han clasificado de la siguiente manera: nada; móviles o aparatos electrónicos; vales de compra; entradas al cine y espectáculos; viajes y estancias; y otros.

8 Cada ítem puede estar compuesto por signos tipográficos (texto) o por imágenes, que a su vez pueden ser fotografías, dibujos o infografías. La clasificación de cada aportación se ha llevado a cabo en base a las siguientes cuatro combinaciones: Sólo texto, Fotografía + Texto, Dibujos / infografías + texto y Dibujos / infografías + foto + texto. 
miento del enlaCe. Este proyecto nació en el año 2006, y supuso un antes y un después en el modelo de participación ciudadana de este periódico. Por lo tanto se tomaron ejemplares (una semana por mes) del año anterior a su implantación (2005) hasta la última renovación de $E l$ Correo, en el año $2010^{9}$.

Además se han llevado a cabo una serie de entrevistas a profesionales de $\mathrm{El} \mathrm{Co}$ rreo $^{10}$.

\section{Contexto de la implantación de «enlaCe» en el diario EI Correo}

Algunos años antes del lanzamiento de enlaCe, el diario El Correo se anticipaba a una era en la que la adaptación de los medios de comunicación a la participación ciudadana se convertiría en uno de los retos del periodismo del siglo XXI. Ya en el año 2000, el periódico decidió renovarse para ofrecer un tipo de periodismo más cercano al ciudadano, tanto a nivel de contenido, ofreciendo una información con mayores dosis de noticias enfocadas a servicios e interés social, como a nivel de participación ciudadana en el propio diario. De hecho, el entonces director del periódico Ángel Arnedo impulsó un cambio a todos los niveles modificando diseño, contenido e incluso la propia organización redaccional. En la actualidad Arnedo opina que fue en aquel cambio donde se gestó el germen de lo que después conoceríamos como enlaCe:

"El inicio del periodismo ciudadano en El Correo comienza con el cambio de $\mathrm{El} \mathrm{Co-}$ rreo 2000. Es bastante revolucionario porque supone una concepción nueva de hacer periodismo. [...] Además cada cierto tiempo hay que hacer algo, si no tienes un cambio te lo tienes que inventar, hay que renovarse". ${ }^{11}$

Los responsables del rotativo vizcaíno consideraron que los periódicos tradicionales en general estaban tremendamente politizados, eran aburridos y bastante contrarios a lo que intuían que un lector esperaba de su diario. Ángel Arnedo opina que los periódicos casi siempre van un poco por detrás de los ciudadanos y a veces les falta ritmo para acompasarse a una sociedad que evoluciona constantemente.

A partir del 22 de marzo del año 2000 se comenzó a publicar lo que sus responsables consideran un diario más acompasado a la sociedad del recién estrenado siglo XXI. Se empezaron a tener en cuenta las nuevas tecnologías, que se introducían a pasos agigantados en las actividades cotidianas de los ciudadanos. El lector tomó un protagonismo mayor. Se le hacían encuestas, se publicaban sus fotografías e incluso se le pedían valoraciones sobre temas de actualidad.

La sección dedicada a la participación ciudadana mantuvo el mismo nombre, "La voz del lector", pero a partir del cambio las vías de contacto se modernizaron. Ya entonces se empezó a impulsar la participación a través de Internet porque el diario pu-

9 En esta última renovación es cuando enlaCe deja de ser el modelo integral de participación a lo largo de todo el diario.

10 Se han realizado entrevistas a Ángel Arnedo, quien fuera director de El Correo en el momento del lanzamiento de enlaCe; a Montse Lluis, impulsora de la idea; al Juan Carlos Martínez, director actual de El Correo, Koldo Dominguez, responsable de enlaCe, etc.

11 Todas las citas del que fuera director de El Correo Ángel Arnedo se han obtenido mediante entrevista presencial 
blicaba la dirección de su Web y un correo electrónico específico (fig.1) para establecer un contacto más inmediato con el lector. Antes del cambio los ciudadanos solamente podían enviar sus cartas y sugerencias a través del correo postal, por fax o llamando al número de teléfono fijo de la centralita del periódico.

A medida que avanzaba la primera década del siglo XXI, la proliferación de medios digitales y gratuitos comenzó a repercutir en la

\section{LA VOZ DEL LECTOR}

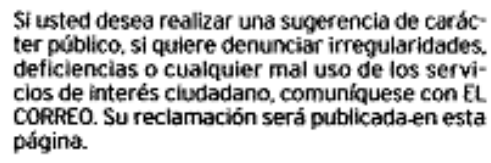

Si usted desea realizar una sugerencia de carácter público. si quiere denunciar irregularidades. deficiencias o cualquier mal uso de los servicios de interés ciudadano, comuniquese con EL CORREO. Su reclamación será publicada-en esta página.

TELÉFONO DEL LECTOR 944111778 Fax: 944734297 Internet: www.eicerreodigitalcom Correo electrónicot vozdeliectorøsib.es
Orección: Pintor Losada, 7, 48004 BULAOO

FIoura 1 difusión de los periódicos tradicionales. Las ventas de los diarios de papel entraban en una escandalosa tendencia a la baja a nivel mundial. Según Ángel Arnedo, lo primero que notaron en España los profesionales del sector fue la pérdida de la lectura del segundo periódico. Hasta entonces, era frecuente que el ciudadano adquiriera un periódico local, el de las farmacias de guardia y las esquelas, y por otro lado como segunda lectura complementaria, un diario nacional. "El segundo diario podía ser El País, La Vanguardia... Internet nos quitó ese segundo periódico. Con Internet perdimos eso. Y no nos hemos recuperado".

Por otro lado, el entonces director del rotativo vizcaíno pensaba que los periódicos seguían estando demasiado politizados y no atraían a la gente joven, lo que le hacía avistar un problema en el relevo generacional de los lectores. También lo pensaba de su propio periódico, a pesar de que había mejorado con el cambio del año 2000. Fruto de estas reflexiones, las bajadas de las difusiones, viendo el panorama de fuera (los periódicos americanos perdían difusión a borbotones) y el avance de las nuevas tecnologías, Ángel Arnedo decidió dar un paso más. A finales del año 2005 empujó a su equipo de redacción hasta la línea de salida de un modelo de periódico con mayor participación de los lectores: "Que el lector sea parte importante del periódico y colabore en la elaboración del diario. Así surgió la idea de enlaCe".

El proyecto fue impulsado por el director del diario quien a su vez confió en la redactora Montserrat Lluis para poner en marcha todas las piezas de la nueva maquinaria participativa. La joven periodista recuerda la intención de ambos de caminar hacia un modelo de prensa participativa en papel:

"Nos dábamos cuenta de que todo el mundo cambiaba menos nosotros. Los periódicos iban para abajo y había que ponerse las pilas. Hasta ahora los periódicos estaban en el centro del universo y los lectores alrededor gravitando. Esto hoy ha cambiado. Son los lectores los que están en el centro, nosotros somos órbitas alrededor de ellos y encima no somos los que más cerca estamos de ellos, lo que nos obliga a buscar nuevas fórmulas y apostar por nuevos contenidos" ${ }^{12}$.

12 Todas las citas de Montserrat Lluis se han obtenido mediante entrevista presencial 
Montse Lluis, que en la actualidad es directora de la secretaría de difusión de medios regionales del grupo Vocento, elaboró toda una filosofía de trabajo alrededor de enlaCe. El propio nombre de la nueva sección fue propuesto por ella misma, lo que Arnedo considera un acierto:

"La idea de llamar enlaCe a la herramienta de participación que se iba a ofrecer al ciudadano fue muy afortunada. Es enlazarse, hacer cadena con el lector. Demuestra la intención de hacer partícipe al ciudadano en todos los aspectos posibles. Lo que hizo Montse fue ir pensando en iniciativas de las que el lector pudiera formar parte, de lo que fuese. Comentando películas de cines, opiniones sobre la televisión, sobre la bolsa...y tuvieron una respuesta casi inmediata. El primer año se llegó a las 100.000 participaciones".

Pero sobre todo, enlaCe sólo tenía sentido en un medio local o multilocal como es El Correo. De hecho, la participación ciudadana que se pretendía fomentar provenía precisamente de hombres y mujeres locales, cuyas preocupaciones e intereses estaban muy ligados al ámbito político, económico y social más cercano. A los ciudadanos frecuentemente les interesan hechos que a veces proceden de la rutina cotidiana, los "temas que proceden de la esfera de lo privado, las noticias que alimentan la páginas de local de los medios" (PEÑALVA, 1996). De hecho, es más fácil crear comunidad y estrechar los vínculos entre la redacción y su audiencia en los medios de ámbito geográfico local o regional. "Forjar vínculos entre el medio y los lectores, así como entre lectores, vivifica el papel de servicio de las ediciones regionales" (LARRONDO, 2009: 114)

"El Correo inicia el lunes 30 de enero la mayor operación de periodismo ciudadano lanzada por un medio en papel". Con estas palabras abría Juan VARELA su blog $P e$ riodistas 21 tres días antes del lanzamiento de enlaCe, en enero del 2006. VARELA explicaba que las áreas como Ciudadanos (local), Economía, Deportes o Vivir y los propios servicios contarían con la participación del público. De ese modo, los lectores podrían enviar informaciones, participar en encuestas, hacer preguntas a la redacción, a las autoridades públicas, a posibles entrevistados, y hasta publicar sus propias críticas de cine, música, televisión, etc.

A partir de entonces, El Correo alojaba una amplia oferta de herramientas participativas para el lector multiplicando su capacidad de generador de contenidos de forma regular. De hecho, casi todas las secciones abrieron espacios para incorporar las contribuciones de los lectores. En unos casos se trataría de comentarios u opiniones remitidos por los ciudadanos de forma esporádica, pero en otros consistiría en espacios fijos, como críticas de cine y televisión o un concurso de Bolsa en las páginas de economía. La mayor parte de las colaboraciones de los lectores se publicarían en la doble página central de enlaCe, donde tendrían cabida artículos, fotografías, felicitaciones, agendas de ocio, contactos o búsquedas de personas concretas, quejas e, incluso, obituarios, remitidos por los lectores. Estas páginas centrales se dedicarían los domingos al público infantil, mediante pasatiempos, chistes y concursos.

\section{Resultados del análisis: Evolución de la participación en enlaCe}

La cantidad de participaciones, el espacio que ocupa cada una de ellas y su contenido han sido eje fundamental de este exhaustivo trabajo de campo. Cada ejemplar analizado ha sido revisado de principio a fin. Todas y cada una de las páginas han sido exa- 
minadas habida cuenta de que el proyecto de participación ciudadana que ofrece $E l$ Correo bajo la denominación enlaCe, no es considerado por el propio diario como una sección cerrada de dos páginas, sino como una filosofía en sí misma que garantiza un modelo de participación integral a lo largo de todo el diario. Así fue hasta la renovación del diario en diciembre del año $2009^{13}$.

El impacto inicial de la implantación de enlaCe genera un incremento de aportaciones ciudadanas publicadas en el diario en todas las categorías analizadas. Por tanto, el primero de los datos que se extraen de este trabajo de campo confirma la realidad

Cantidad participaciones por dia

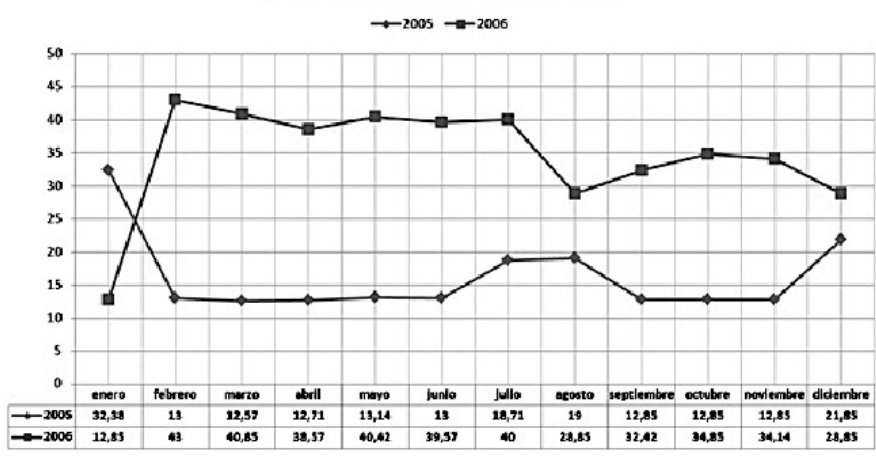

Figura 3 (Elaboración propia) que a simple vista resulta obvia: el número de participaciones de los lectores y el espacio que estas ocupan dentro del diario El Correo aumenta inicialmente a partir de la existencia de enlaCe. La media de participaciones diarias durante el año 2005, previo a la implantación de enlaCe, es de 16,24 aportaciones dia-

rias. Esto es, El Correo ha ofrecido un espacio de expresión para 16 de sus lectores cada día. En el año 2006 esta cifra asciende a 34,53 participaciones diarias, de lo que podemos extraer el primer dato concluyente: Gracias a enlaCe el número de lectores que tiene la oportunidad de alzar su voz, en cualquiera de las formas que sea, se multiplica por dos.

Pero a la larga, la tendencia clara del número de lectores que aporta algún
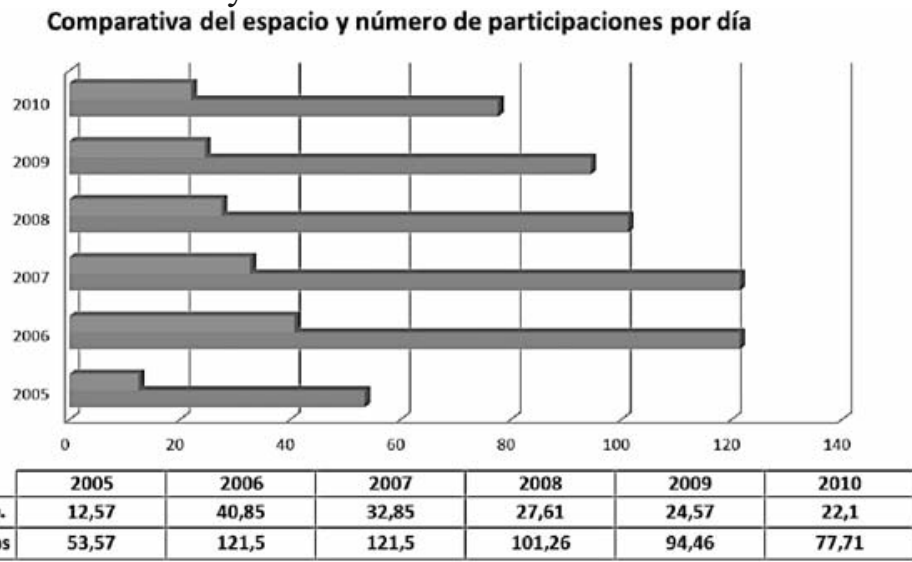

Figura 4 (Elaboración propia)

${ }_{13}$ A finales del año $2009 \mathrm{El}$ Correo fue sometido a un rediseño general que afectó a todo el periódico (y al resto de periódicos del grupo Vocento), lo que repercutió de forma directa y notable en enlaCe, el objeto de estudio de esta investigación. La aplicación del análisis hemerográfico mediante la ficha en esta etapa fue considerada fundamental para entender hasta qué punto se reduce a partir de este cambio la participación ciudadana, y cuál de los tipos de participación se reduce de manera más contundente. 
tipo de contenido a $\mathrm{El}$ Correo, y por consiguiente, el espacio que este otorga a estas participaciones es a la baja.

El gráfico muestra (fig.4) que en el primer año de enlaCe, 2006, se registran en marzo unas 40 unidades participativas por día, frente a las 12 unidades registradas un año antes. Los siguientes años esta cantidad va disminuyendo hasta encontrarnos en el año 2010 con una media de 22 ítems por día. Si bien es cierto que la cantidad no se reduce hasta los términos conocidos en el año 2005, no es menos cierto que la bajada respecto al primer año de enlaCe roza el 50\%.

Sin embargo, tal y como se puede comprobar en la figura 5 , el porcentaje de contenido del periódico generado por lectores en la etapa renovada de $\mathrm{El} \mathrm{Co}$ rreo no es menor que en el año anterior (2009) y queda muy cerca del de dos años antes (2008). Lo cierto es que, con la renovación del diseño, contenido y filosofía del periódico, el número

\section{Contenido de El Correo generado por lectores}

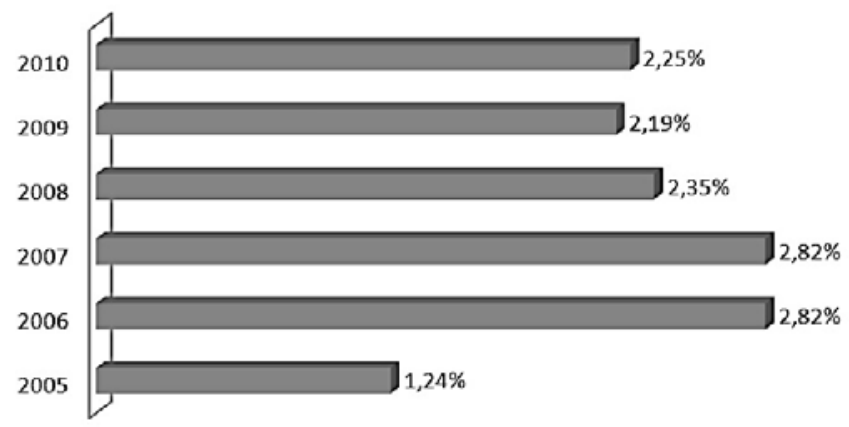

Figura 5 (Elaboración propia)

de páginas se reduce considerablemente. Pasa de una media de 86 páginas a una media de 70 , y frente a este recorte, enlaCe pierde cantidad de unidades participativas, pero mantiene su proporción de espacio.

Las aportaciones de interés general se mantienen del mismo modo, aparentemente, en el año 2010 respecto al año anterior. Incluso se puede decir que aumentan levemente en cantidad de unidades por semana. Aun así, habría que comprobar si todos los tipos de aportación de esta categoría mantiene la misma tendencia.

Evolución de los tipos de aportación de Interés General

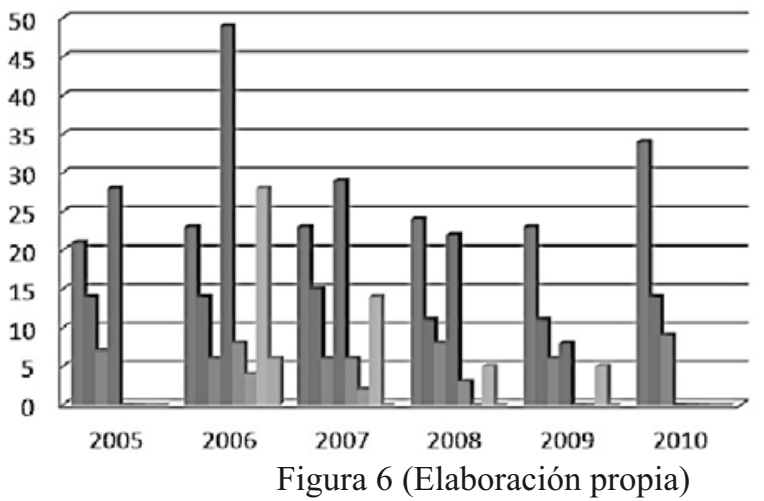

Clasificando las aportaciones de interés general en los siete dis- TIPO A tintos tipos posibles ob口TIPO B tenemos datos $\square$ TIPOC concluyentes (fig.6). - TIPO D Las aportaciones de - tIPOE tipo A (las cartas al di- TIPOF rector) se mantienen constantes todos los años, e incluso suben considerablemente a partir de la renovación del El Correo en el año

2010. Lo cierto es que en esta nueva etapa se ha ampliado el espacio dedicado a las cartas al director. La media durante los años 2005, 2006, 2007, 2008 y 2009 oscilaba 
las 23 cartas a la semana y en el 2010 obtenemos una media de 34 cartas por semana. Dicho de otro modo, la media ha pasado de 3,2 cartas al día a 4,85 cartas al día.

Observamos cómo las aportaciones de tipo B (cartas de ámbito cercano) y C (fotografías de interés general) se mantienen más o menos estables, y son las aportaciones de tipo $\mathrm{D}$ (respuestas de los lectores a encuestas planteadas por el diario) las que sufren el cambio más brusco con la llegada de enlaCe y su posterior reajuste. De hecho, las encuestas, suben en cantidad de forma destacable el primer año de la implantación de enlaCe. Pasan de registrarse 28 aportaciones de este tipo a la semana (4 al día), a 49 por semana ( 7 al día), para ir bajando poco a poco hasta desaparecer, ya que, en el año 2010 no se registra ninguna aportación de este tipo.

Llama la atención que el resto de aportaciones, las de tipo E (pregunta-respuesta experto), $\mathrm{F}$ (críticas al diario), G (críticas culturales) y $\mathrm{H}$ (respuestas masivas en porcentaje) también desaparecen por completo. Este tipo de participaciones eran ya escasas en los últimos años. Su desaparición compensa la subida de las aportaciones de tipo A.

En cuanto a las aportaciones de ámbito privado, estas muestran el siguiente panorama. A raíz del lanzamiento de enlace suben de manera considerable, y la tendencia durante los años posteriores ha sido una ligera subida, al margen de que en marzo del 2008 bajara la media semanal. Sin embargo, el análisis por separado de cada tipo de aportación de ámbito privado destapa otros datos que no se pueden obviar. Sí existen tendencias a las baja y al alza según el tipo de participación. Las de tipo A, que corresponden a las cartas de ámbito privado, se quedan como estaban antes de enlaCe, con una media de 3-4 aportaciones por semana. Las de tipo B, que corresponden a las fotos de ámbito privado de los lectores, aumentan cada año y mantienen esa misma tendencia en el año 2010, y las de tipo C, o los dibujos, desaparecen, por lo menos de forma generalizada o periódica, al margen que de manera puntual se organicen campañas navideñas o de otro tipo que impliquen este tipo de participación.

№ aportaciones por semana de Ámbito Privado

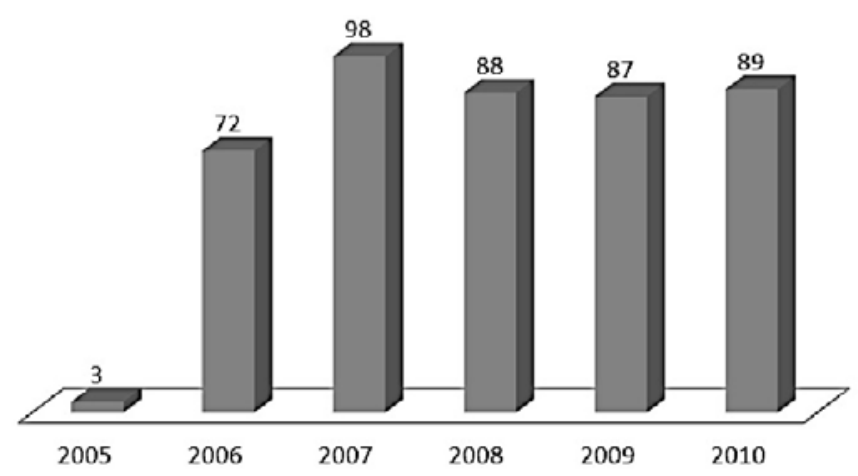

Figura 7 (Elaboración propia)

En lo que respecta a las aportaciones enmarcadas en la categoría 3, las correspondientes a las informaciones de servicio suministradas por los lectores, el máximo auge se registra en el primer año del lanzamiento de enlace. Posteriormente las participaciones de este tipo sufren una caída considerable hasta mantenerse en una media de una aportación por semana.

En el caso de las aportaciones de ámbito privado elevadas a interés general la bajada también es destacable. Es más, tras la renovación de El Correo a finales del 2009 llega casi a desaparecer, ya que se suprimen los espacios que a esta categoría dedicaba enlaCe bajo títulos como "la familia del mes", "sueños confesables" o "mi colección". 
Las aportaciones correspondientes a la categoría 5, las de concursos y retos, desaparecen completamente a partir del reajuste de enlaCe en la etapa renovada del periódico. La novedad en esta categoría la encontramos en los ejemplares del 2007, en los que se detecta un nuevo concurso gastronómico de recetas de cocina, y en los correspondientes a 2008 y 2009, en los que registra el concurso o reto para los lectores de elaborar una portada para el periódico.

\section{Conclusiones}

1. El nacimiento de enlaCe tiene como objetivo acercarse al ciudadano, hacerle sentir parte del periódico. Esto ha sido así desde el cambio de El Correo en el año 2000, pero sobre todo a raíz de la incorporación de enlaCe. Se cumple la hipótesis que afirmaba que la adaptación de El Correo al nuevo modelo participativo se hizo de forma paulatina. Además de la información obtenida a través de las entrevistas, hemos observado cómo en el período del 2005 ya se lanzaban campañas puntuales que incrementaban la participación ciudadana: se fomentaba el envío de dibujos en las navidades, la publicación de fotos de las vacaciones de verano o las encuestas. Desde la incorporación de enlaCe los periodistas aparecen con foto, dispuestos a mostrar su cara para resultar más familiares. Publican su dirección de e-mail, que de algún modo demuestra su intención de intercambiar opiniones con los lectores. Por lo tanto, la manera de acercarse al ciudadano, y hacer un periodismo más social es:

- Mostrarse cercanos a los lectores, con nombres y apellidos. Con cara. En definitiva, ofrecer un periódico que está dispuesto a escuchar. No mostrarse como un ente superior infranqueable.

- Ofrecer espacios y herramientas reales a los lectores para garantizar su participación activa en la elaboración del contenido del periódico. Esto es, incidir en la idea de Montse Lluis de bajar la redacción del pedestal desde donde monopolizaba y manejaba la información sin contar con los ciudadanos.

2. Con la llegada de enlaCe el espacio que El Correo dedica a aportaciones de los lectores aumenta de manera importante el primer año. Las contribuciones de los ciudadanos publicadas por el diario pasan de un 1,69\% (durante el año 2005) a ocupar un 2,64\% (2006) respecto al contenido total del diario. Además, el número de contribuciones diarias se duplica, pasando de una media de 16,24 a 34,53 aportaciones publicadas de media diaria. Dicho de otro modo, durante el año 2005 son 16 las personas que consiguen publicar algo en cada ejemplar de El Correo, frente a 34 que lo hacen cada día durante el año 2006.

3. Con enlaCe, El Correo opta por fidelizar a la audiencia ofreciéndole la opción de expresarse sobre temas amables o muy pegados a la realidad más cercana del mismo. Las aportaciones de interés general más numerosas (no las que más espacio ocupan) durante el primer año de enlaCe son las respuestas de los lectores a las encuestas planteadas por el diario. Estas preguntas que plantea el periódico suelen estar relacionados con debates de actualidad o con asuntos de la realidad local. Desde la normativa del tabaco en los bares, hasta la suciedad en las calles de Bilbao, por poner dos ejemplos.

4. EnlaCe funciona como una red social de papel para los lectores de El Correo. Lo fue así en sus inicios y lo sigue siendo en la actualidad. Hoy por hoy, tanto los lec- 
tores que envían sus aportaciones de interés general (sobre todo cartas de opinión) como los que envían aportaciones de ámbito privado (fotos de cumpleaños, familiares, felicitaciones, etc.) no reciben ningún tipo de compensación a cambio. Los lectores encuentran la recompensa a su participación en la propia publicación de sus aportaciones. Por lo tanto, el objetivo de las personas que envían sus opiniones es que tanto los profesionales del periódico como la audiencia les escuchen. Y el objetivo de aquellos que envían las fotos de sus hijos, nietos, abuelos, comuniones, bodas, cumpleaños... es que sus seres queridos puedan ser vistos por la red social de conocidos y no tan conocidos que leen el periódico. Más aun teniendo en cuenta que una gran parte de la audiencia de este diario responde a un perfil de lector no familiarizado con otras redes sociales digitales y los recursos de la Web 2.0.

5. Por encima de los intentos por buscar nuevas fórmulas participativas en su edición de papel, las "Cartas al director" se erigen como las reinas de la participación ciudadana en El Correo. Y es que han salido reforzadas de esta experiencia. Tras el rediseño del periódico, todos los espacios dedicados a la participación ciudadana se han visto reducidos, excepto el de las "Cartas al director", que ha aumentado.

\section{Referencias bibliográficas}

ARMENTIA, Ignacio (2009):"La Evolución del periodismo participativo en Internet”, en PÉREZ, P.; RIVAS, P.; GELADO, R. (coord.): Estudios de Periodística XIV. Salamanca, Universidad Pontificia de Salamanca, p. 19-29

BABBIE, Earl (1999): Técnicas de la investigación social. México, Thompson.

BARDIN, Laurence (1986): Análisis del contenido. Madrid, Akal

GARCÍA DE TORRES, Elvira et al (2009): "La agenda de los usuarios: un análisis de la participación en la página principal y la sección local de elpais.com y 20minutos.es" en PÉREZ, P.; RIVAS, P.; GELADO, R. (coord.): Estudios de Periodística XIV. Salamanca: Universidad Pontificia de Salamanca, p. 287-299

HOYO, Mercedes del; OLMO BARBERO Jesús del (2009): "Prensa gratuita, canal de participación ciudadana" en PÉREZ, P.; RIVAS, P.; GELADO, R. (coord.): Estudios de Periodística XIV. Salamanca, Universidad Pontificia de Salamanca, p. 317-323

JIMENO LÓPEZ, Miguel Angel (2009): “Los retos del nuevo periodismo ¿Para qué sacamos ciudadanos en el diario si no pensamos en ellos?" en PÉREZ, P.; RIVAS, P.; GELADO MARCOS, Roberto (coord.): Estudios de Periodística XIV. Salamanca, Universidad Pontificia de Salamanca, p. 206-215

LARRONDO URETA, Ainara (2009): "La estrategia editorial del diario papel en el mercado del periodismo online y ciudadano. El caso de «enlaCe»" en PÉREZ, P.; RIVAS, P.; GELADO, R. (coord.): Estudios de Periodística XIV. Salamanca, Universidad Pontificia de Salamanca, p.107-115

LÓPEZ HIDALGO, Antonio; FERNÁNDEZ BARRERO, María Ángeles (2009): "Periodismo ciudadano. Los modelos de participación de la audiencia y sus grados 
de dependencia del periodismo profesional" en PÉREZ, P.; RIVAS, P.; GELADO, R. (coord.). Estudios de Periodística XIV. Salamanca, Universidad Pontificia de Salamanca, p.187-194

MAYORAL SÁNCHEZ, Javier (2009): "Los trabajos del ciudadano-periodista" en PÉREZ, P.; RIVAS, P.; GELADO, R. (coord.). Estudios de Periodística XIV. Salamanca, Universidad Pontificia de Salamanca, p. 365-371

MESO, Koldo; PALOMO, María Bella (2009): "Análisis del periodismo participativo elaborado en los ciberdiarios de Vocento" en PÉREZ, P.; RIVAS, P.; GELADO, R. (coord.). Estudios de Periodística XIV. Salamanca, Universidad Pontificia de Salamanca, p.253-262

PASTOR, Lluis (2009): Teoría De las cartas al director. La gestión periodística del público I. Barcelona, UOC

PEÑALVA, José Luis (1996): Prensa regional y nuevas vías de acercamiento al lector. Bilbao, Universidad del País Vasco

RUIZ, Carlos; MASIP, Pere; MICÓ, Josep Lluis.; DÍAZ NOCI, Javier y DOMINGO, David (2010): "Conversación 2.0. y democracia. Análisis de los comentarios de los lectores en la prensa digital catalana" en: Comunicación y Sociedad, vol. XXIII, n. 2, pp.7-39.

RUIZ OLABUENAGA José Ignacio; ISPIZUA, María Antonia (1989): La descodificación de la vida cotidiana. Métodos de investigación cualitativa. Bilbao, Universidad de Deusto.

VARELA, Juan (2006): "El Correo se abre al periodismo ciudadano" en Periodistas 21, disponible en: http://periodistas21.blogspot.com/2006/01/el-correo-se-abreal-periodismo_27.html [Consulta: 15-3-2009]

VISAUTA, Bienvenido (1989): Técnicas de Investigación Social. I: Recogida de datos. Barcelona: Promociones y Publicaciones Universitaria (PPU) 